\title{
Syndesmotic Ankle Sprains
}

Jack Ransone, PhD, ATC; Phill Vardiman, MS, ATC; and Kenneth Smith, DO • Oklahoma State University

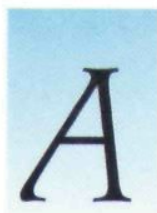

NKLE INJURIES are among the most common injuries in sports. Those involving the talo- and tibiofibular ankle ligaments are among the most common and debilitating afflictions of the lower leg. Syndesmosis ankle sprains are uncommon and difficult to diagnose because of the subtle anatomical changes involved. When the injury goes undetected, the outcome can be persistent pain and instability that can lead to further injury. The structural damage resulting from syndesmosis sprains is comparable to ankle-joint fractures in terms of time to heal from surgery and the length of disability. The major function of the syndesmosis is to maintain the articulation between the tibia and fibula. The force applied to the ankle that causes a syndesmosis injury is usually forced external rotation, which, with the foot in a neutral position, disrupts mostly the anterior syndesmosis structures. When combined with dorsi- or plantar flexion, forced external rotation of the foot can damage the lateral and medial structures of the foot, as well as the syndesmosis.

\section{The Case}

During a regular-season game, a 21 -year-old Division-I college football player reported to the sidelines complaining of pain on the anterolateral portion of his left ankle. He demonstrated the mechanism of injury as both plantar flexion and inversion of his ankle and reported feeling a pop in his ankle and immediate pain. Physical examination revealed mild edema on the anterolateral surface of the ankle. Palpation revealed no obvious deformity or subluxation. Manual testing bilaterally proved to be within normal limits and graded at 4/5 in dorsiflexion. Anterior drawer and talar tilt were positive for pain, showing slight laxity with a strong end point. Radiographs revealed no fracture or obvious joint diastasis (Figure 1). The athlete returned to activity with ankle taping and had no complications after approximately 3 weeks of rest and rehabilitation. Five months after the initial injury, he injured his ankle during the off season. Immediate care consisted of ice packs, compression wraps, and elevation to reduce mild swelling. The athlete was released to full weight bearing but with activity limited because of pain.

When the athlete returned to activity 2 weeks after the second ankle sprain, he continued ankle exercises with mild pain in the superior aspect of the ankle joint. After 1 month, the pain progressed and moved up the shaft of his lower leg. Radiographs revealed the beginning of heterotropic bone formation on the interosseous membrane between the tibia and fibula (Figure 2). The athlete was released to compete to the level of his pain tolerance. One week later, he received a direct blow to the area. Orthopedic examination identified maturation of a heterotropic bone formation in the interosseous membrane approximately $10 \mathrm{~cm}$ from the articulation of the tibia and fibula (Figure 3). An anklefoot orthosis was prescribed for 3 weeks to help re-

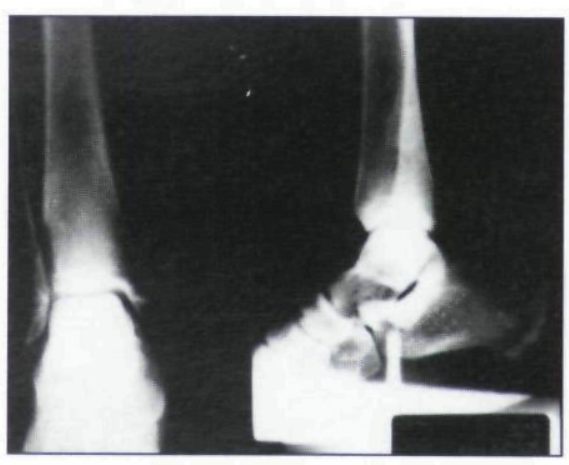

Figure 1 Initial X ray.

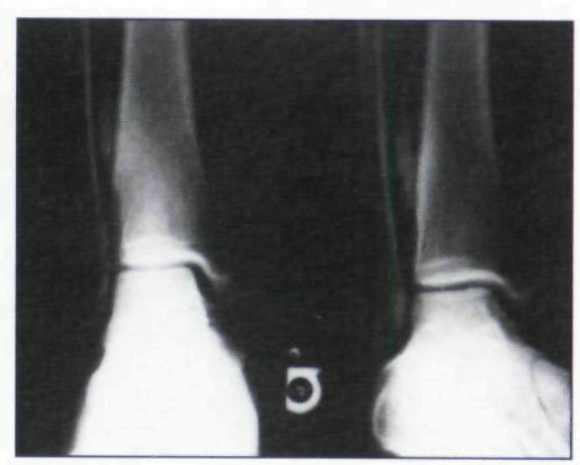

Figure $2 \mathrm{X}$ ray 1 month postinjury revealing heterotropic bone formation on the interosseous membrane.

- 2001 Human Kinetics AIT 6(5), pp. 48-49 


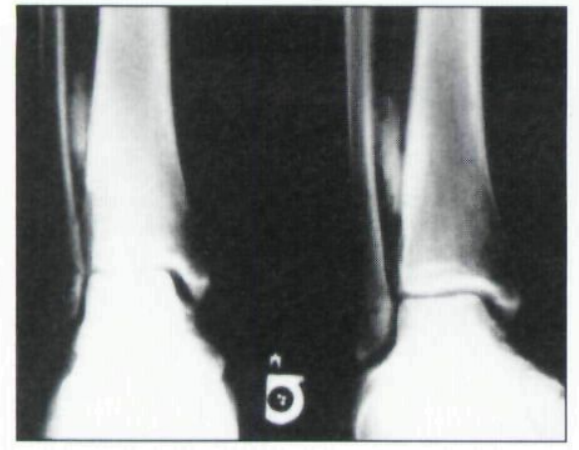

Figure $3 \mathrm{x}$ ray 5 weeks postinjury revealing maturation of heterotropic bone formation.

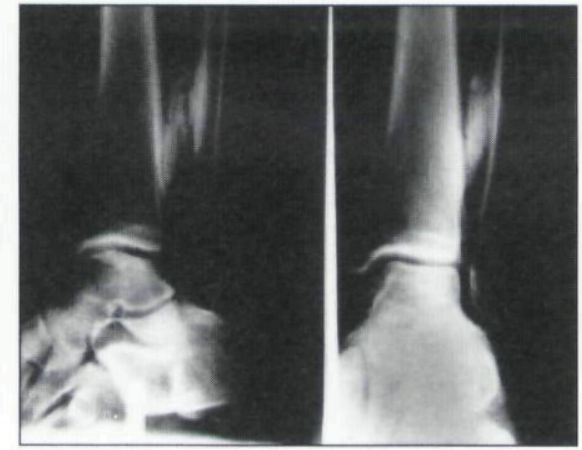

Figure $4 \quad \mathrm{X}$ ray 11 months postinjury showing significant changes in calcification.

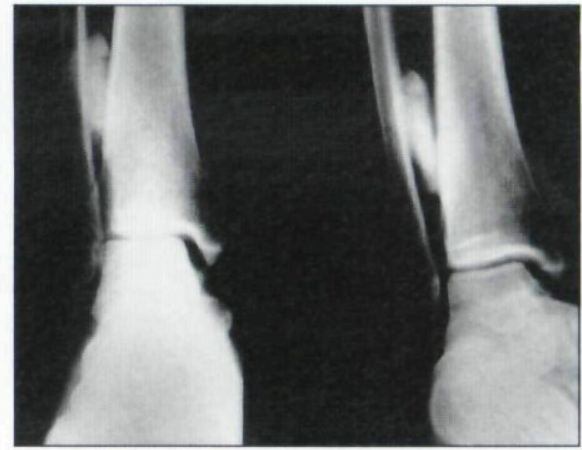

Figure $5 \times$ ray 1 year postinjury showing increased size of calcification. duce pain and maintain the athlete's ability to bear weight. Two weeks later, the athlete showed significant improvement in stability and stated that the pain was dramatically reduced. 2-month-postinjury $\mathrm{X}$ rays confirmed stable heterotropic formation. At the beginning of the next regular season, 11 months after the initial injury, $\mathrm{X}$ rays revealed a change in the calcification (Figure 4). Immobilization for 3 weeks with gradually increasing activity was prescribed. Subsequent $X$ rays 1 week later revealed the beginning of maturation and increase in size of the calcification (Figure 5). A Bill Berringer brace/orthosis (Figure 6) was prescribed to prevent excessive plantar and dorsiflexion. The athlete competed during the regular season and postseason with minimal difficulty and moderate pain.

\section{Discussion}

Syndesmosis injuries without fracture are fairly uncommon in athletics. They affect the stability of the ankle mortise with disruption of the anterior, posterior, and transverse tibiofibular ligaments and the interosseous membrane. In the case of a ligamentous sprain to the ankle, complaints of lower leg pain that extends up the shaft of the lower leg along the interosseous membrane should be watched for. External rotation involved in the mechanism of injury is often a red flag to evaluate for possible syndesmosis injury. Inversion or eversion ankle sprains should not be ruled out as a possible cause of syndesmosis injury. Gait analysis typically reveals an inability to bear weight or plantar flex the affected side. Signs of syndesmosis injury might include prolonged recovery, recurrent ankle sprains, and heterotropic bone formation resulting from laxity in the injured syndesmosis ligaments. Any of these signals, once identified, would indicate the need for a complete evaluation that might include stress $\mathrm{X}$ rays, CT scan, and MRI. Follow-up stress films should be taken in forced abduction and external rotation to detect a widening of the ankle mortise. These films might reveal heterotropic bone formation, which is common during syndesmosis injury but takes 46 weeks to appear on $\mathrm{X}$ ray. With identification of a widened mortise, laxity of syndesmosis ligaments,

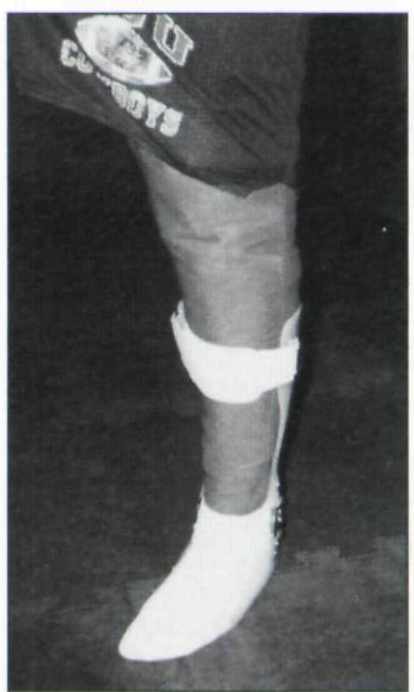

Figure 6 Bill Berringer brace/orthosis. or heterotropic bone formation, the athlete can be informed of the prognosis.

Athletic trainers and therapists should be aware of syndesmosis sprains and their pathology. When these injuries are overlooked as general ankle injuries, conservative management does not resolve the symptoms. Syndesmosis sprains are associated with recovery times at least twice as long as those for common lateral ankle sprains. Treatment should include a period of non-weight-bearing to allow soft-tissue healing. Management of syndesmosis sprains can possibly include recurrent ankle sprains and surgical intervention or external bracing to correct instability of the ankle mortise. I

Jack Ransone is a professor and director of athletic training education at Oklahoma State University.

Phill Vardiman is an assistant athletic trainer at the University of Arkansas in Fayetteville, where he is currently pursuing a doctoral degree. Kenneth Smith is team physician in the Department of Athletics at Oklahoma State University in Stillwater. 
Copyright of Athletic Therapy Today is the property of Human Kinetics Publishers, Inc.. The copyright in an individual article may be maintained by the author in certain cases. Content may not be copied or emailed to multiple sites or posted to a listserv without the copyright holder's express written permission. However, users may print, download, or email articles for individual use. 